\title{
Application de l'analyse isotopique par spectrométrie de masse et sonde ionique de l'oxygène des émeraudes naturelles
}

\author{
G. Giuliani ${ }^{1,2}$, M. Chaussidon ${ }^{2}$, C. France-Lanord ${ }^{2}$, C. Rollion ${ }^{2}$, D. Mangin ${ }^{2}$ et P. Coget ${ }^{2}$ \\ ${ }^{1}$ IRD, Institut de Recherche pour le Développement, Paris, France \\ ${ }^{2}$ CRPG-CNRS, UPR 9046, BP. 20, 54501 Vandouvre-lès-Nancy Cedex, France
}

Simple and non-destructible methodologies for the authentication of gems like emeralds are generally not efficient enough for confirming geographical and geological origins [1]. This paper deals with the use of isotopic methodologies for emeralds authentication. An isotope ratio mass spectrometer coupled with an ionic probe allows to measure ${ }^{18} \mathrm{O} /{ }^{16} \mathrm{O}$ ratios from oxygen which is the main constitutive element of these gems $[2,3]$. The isotopic study of 62 different deposits allowed to draw up the map of oxygen isotopic ratios and to identify the origin of most of the high-grade emeralds marketed all over the world.

\section{L'émeraude et les isotopes de l'oxygène}

L'émeraude, variété chromifère et vanadifère du béryl, de formule idéale $\mathrm{Be}_{3} \mathrm{Al}_{2} \mathrm{Si}_{6} \mathrm{O}_{18}$, est plus rare que le diamant et peut avoir une valeur commerciale mille fois supérieure à celle de l'or. La Colombie est le premier producteur mondial d'émeraude. De 1990 à 1995, le volume de gemmes colombiennes exportées a été de 23 millions de carats, soit environ $60 \%$ de la production mondiale [3]. Les autres producteurs sont la Zambie (15\%), le Brésil (10\%), la Russie (5\%), Madagascar (3\%), le Zimbabwe (2\%) et d'autres pays (5\% dont l'Afghanistan et le Pakistan). Les émeraudes de Colombie sont estimées pour leur qualité exceptionnelle au même titre que celles d'Afghanistan, de Zambie et de Russie.

L'oxygène est le constituant chimique le plus abondant de la croûte terrestre et le constituant majeur de l'émeraude. En effet, l'oxygène structural représente 45 poids $\%$ ou 65 atome $\%$ du minéral. L'oxygène possède trois isotopes stables, respectivement ${ }^{16} \mathrm{O}=99,756 \%,{ }^{17} \mathrm{O}=0,039 \%$, ${ }^{18} \mathrm{O}=0,205 \%$. On peut rappeler que les isotopes d'un même élément chimique possèdent le même numéro atomique $\mathrm{Z}$ (même nombre de protons dans leur noyau) mais une masse atomique A différente (nombre de neutrons différent). La composition isotopique, rapport d'abondance d'un isotope lourd (et rare : ${ }^{18} \mathrm{O}$ ) sur un isotope léger (et abondant : ${ }^{16} \mathrm{O}$ ) est généralement exprimée par l'écart « $\delta$ » (exprimé en pour mille, \%o) entre les rapports isotopiques de l'échantillon et d'un standard,

soit :

$$
\delta^{18} \mathrm{O}=\left[{ }^{18} \mathrm{O} /{ }^{16} \mathrm{O}_{\text {échantillon }} /{ }^{18} \mathrm{O} /{ }^{16} \mathrm{O}_{\text {standard }}-1\right] \times 1000
$$


Pour l'oxygène, le standard international correspond à l'eau de mer moyenne et est appelé SMOW (Standard Mean Ocean Water). Les rapports isotopiques de l'oxygène ${ }^{17} \mathrm{O} /{ }^{16} \mathrm{O}$ et ${ }^{18} \mathrm{O} /{ }^{16} \mathrm{O}$ ne varient pas indépendamment : dans toutes les roches terrestres, ils sont liés par la loi de discrimination de masse selon l'équation $\delta^{17} \mathrm{O}=0.52 \times \delta^{18} \mathrm{O}$ [4]. De plus, les compositions isotopiques de l'oxygène sont indépendantes de l'âge puisque les trois isotopes de l'oxygène sont des isotopes stables ne provenant pas de la décroissance radioactive d'autres éléments. Par contre, des variations importantes de ces compositions isotopiques (obéissant à la loi de discrimination de masse) sont produites lors des processus géologiques faisant ainsi du rapport ${ }^{18} \mathrm{O} /{ }^{16} \mathrm{O}$ un traceur de choix de ces processus. Ainsi, un échantillon qui possède un $\delta^{18} \mathrm{O}$ de $+10 \%$ ost enrichi en ${ }^{18} \mathrm{O}$ de $10 \%$ o par rapport au standard. À titre d'exemple, les compositions isotopiques des roches granitiques, des roches mafiques-ultramafiques et des calcaires sont comprises respectivement, entre +7 et $+13 \%$, $+5,5$ et $+8 \%$ o +20 et $+30 \%$. La différence relative de masse entre deux isotopes (quelques pour cent), fait que lors de réactions chimiques ou de changements de phase, ils ne se transfèrent pas toujours équitablement entre les produits de départ et d'arrivée. Ce fractionnement isotopique obéit aux lois thermodynamiques d'équilibre isotopique et dépend essentiellement de la température.

\section{Les techniques d'analyse des isotopes de l'oxygène de l'émeraude}

Deux techniques différentes sont utilisées pour l'analyse de l'oxygène :

(1) la première est destructive et correspond à la méthode classique d'extraction de l'oxygène [5]. Une quantité de 2 à $4.10^{-3} \mathrm{~g}$ d'émeraude est placée sous vide dans un tube à réaction en nickel. L'échantillon est oxydé à chaud par un excès gazeux de pentafluorure de brome $\left(\mathrm{BrF}_{5}\right)$. La réaction libère la totalité de l'oxygène structural qui est ensuite transformé en gaz carbonique sur un barreau de graphite porté à une température de $750{ }^{\circ} \mathrm{C}$. Le gaz est purifié et mesuré au manomètre avant d'être collecté dans un tube échantillon. La composition isotopique du gaz carbonique est analysée sur un spectromètre de masse à gaz et la reproductibilité des mesures est de 0,2\%o. Cette technique nous a permis d'élaborer une carte d'identité isotopique ${ }^{18} \mathrm{O} /{ }^{16} \mathrm{O}$ de l'émeraude $[2,3]$.

(2) Grâce à la première technique, nous avons pu calibrer l'analyse isotopique de l'oxygène sur une sonde ionique IMS 1270 à multicollection au C.R.P.G. (CNRS de Vandœuvre). Cette deuxième technique est considérée comme non destructive. En effet, elle permet de mesurer directement sur une surface polie (pierre facetée par exemple) la composition isotopique de l'échantillon. L'émeraude est placée sous vide et elle est bombardée par un faisceau d'ions primaires (source césium produisant un faisceau primaire de 0,1 à $0,2 \mu \mathrm{m}$ de diamètre) dont la foca- lisation détermine le diamètre de la zone analysée (de 0,2 à $400 \mu \mathrm{m}$ ). Les molécules arrachées à l'échantillon (sur un cratère de quelques microns de diamètre et quelques dizièmes de microns de profondeur ; Fig. 1a) sont triées par des secteurs électrostatique et magnétique puis analysées par des détecteurs qui comptent l'intensité des différentes masses. La sonde ionique possède 7 détecteurs d'ions lesquels permettent de mesurer plusieurs isotopes en parallèle et de se rapprocher de la précision analytique des spectromètres de masse classiques. La figure $1 \mathrm{~b}$ compare les résultats préliminaires d'étalonnage des standards d'émeraudes analysés par la technique classique avec 2 à $4.10^{-3} \mathrm{~g}$ d'échantillon détruits et par la sonde ionique en monocollection avec seulement $2.10^{-11} \mathrm{~g}$ consommés.

\section{La carte d'identité isotopique ${ }^{18} \mathrm{O} /{ }^{16} \mathrm{O}$ des émeraudes naturelles}

Les valeurs des $\delta^{18} \mathrm{O}$ obtenues sur les émeraudes sont comprises entre $+6,2$ et $+24,7 \%$ o (Fig. 2). Pour chaque gisement, les valeurs des $\delta^{18} \mathrm{O}$ sont cohérentes et représentatives depuis l'échelle d'une veine minéralisée jusqu'à celle d'un district minier. En effet, pour le gisement colombien d'Oriente (District minier de Chivor), la variation enregistrée sur 12 cristaux d'émeraude, prélevés dans une même veine à carbonates, ne dépasse pas 0,4\%o. De même, à l'échelle du district minier de Carnaíba au Brésil, les variations enregistrées pour les émeraudes provenant de 7 mines distinctes sont insignifiantes $\left(\delta^{18} \mathrm{O}=+9,0 \pm 0,1 \% o, n=7\right.$ mines). Ces résultats montrent que le $\delta^{18} \mathrm{O}$ mesuré sur chaque cristal constitue une signature caractéristique de l'origine de l'émeraude.

Trois groupes de valeurs des $\delta^{18} \mathrm{O}$ sont distingués (Fig. 2) :

(1) le premier groupe, compris entre $+6,2$ et $+7,9 \%$, est défini par les émeraudes brésiliennes du Quadrilatère Ferrifère (gisements d'Itabira-Mina Belmont et de Capoeirana, État du Minas Gerais), d'Autriche (Habachtal), d'Australie (Poona) et du Zimbabwe (Sandawana) ;

(2) le second groupe, défini par l'intervalle +8 à $+12 \%$, concerne la plupart des gisements étudiés, notamment ceux de Zambie (Miku), Tanzanie (Sumbawanga), Russie (Oural), Madagascar (Morafeno), Pakistan (Khaltaro), Inde (Rajhastan) et Brésil (Carnaíba et Socotó) ;

(3) le troisième groupe, caractérisé par des valeurs en $\delta^{18} \mathrm{O}$ supérieures à $+12 \%$, inclut les gisements de Colombie, de Santa Terezinha de Goiás (Brésil ; $\delta^{18} \mathrm{O}=+12,2 \pm$ $0,1 \%, n=4)$, d'Afghanistan (Kendjt, Kalat, Gujari, Shabaki ; $\delta^{18} \mathrm{O}=+13,5 \pm 0,1 \%$, $n=5$ ) et du Pakistan (district de Swat-Mingora ; $\left.\delta^{18} \mathrm{O}=+15,7 \pm 0,1 \% o, n=9\right)$. En Colombie, les émeraudes de la zone orientale (districts de Gachalá et de Chivor) possèdent des $\delta^{18} \mathrm{O}$ plus légers $\left(\delta^{18} \mathrm{O}\right.$ $=+16,8 \pm 0,1 \%$ o, $n=27$ ) que ceux de la zone occidentale (districts de Muzo, Coscuez, Peña Blanca, Yacopí ; $\delta^{18} \mathrm{O}=$ $+21,2 \pm 0,4 \% o, n=17)$. En définitive, les $\delta^{18} \mathrm{O}$ des éme- 


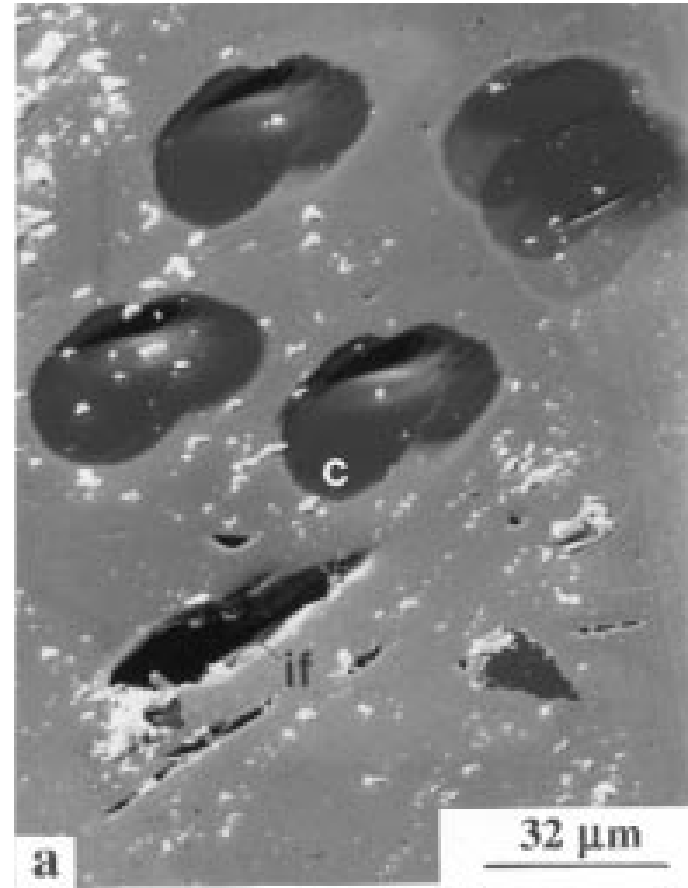

Figure 1a. Aspect tridimensionnel des cratères (c) formés à la surface d'une émeraude à la suite d'une analyse à la sonde ionique ims 1270. Observation au microscope electronique à balayage. La dimension des cratères peut être comparée à celle d'une cavité d'inclusion fluide (if) piégée par le cristal. (Photo A. Kholer, Université Henri Poincaré, Nancy).

raudes de ce troisième groupe sont très contrastés et les intervalles de valeur distincts. Ainsi, leur rapport ${ }^{18} \mathrm{O} /{ }^{16} \mathrm{O}$ peut être utilisé comme une propriété exclusive d'authentification.

\section{La carte isotopique ${ }^{18} \mathrm{O} /{ }^{16} \mathrm{O}$ des émeraudes et ses applications}

\section{Application à l'industrie des gemmes}

Les rapports isotopiques ${ }^{18} \mathrm{O} /{ }^{16} \mathrm{O}$ obtenus sur l'ensemble des échantillons étudiés nous permettent de dépister l'origine des émeraudes naturelles de qualité supérieure, trouvées dans le commerce des pierres précieuses (Fig. 2) : émeraudes provenant du Quadrilatère Ferrifère au Brésil (Capoeirana, Mine Belmont : $\delta^{18} \mathrm{O}=+6,8 \pm 0,4 \%$ ), de Colombie (zone orientale : $\delta^{18} \mathrm{O}=+16,8 \pm 0,1 \%$; zone occidentale : $\delta^{18} \mathrm{O}=+21,2 \pm 0,5 \%$ ) , de Santa Terezinha de Goiás au Brésil $\left(\delta^{18} \mathrm{O}=+12,2 \pm 0,1 \%\right.$ ) , du Zimbabwe (Sandawana : $\delta^{18} \mathrm{O}=+7,5 \pm 0,5 \%$ ), de Zambie (Miku, $\delta^{18} \mathrm{O}=+10,1 \pm$ $0,3 \%$ ) , d'Afghanistan $\left(\delta^{18} \mathrm{O}=+13,5 \pm 0,1 \%\right.$ o $)$ et $\mathrm{du}$ Pakistan (Swat : $\delta^{18} \mathrm{O}=+15,7 \pm 0,1 \%$ ). Cependant, les émeraudes de Carnaíba (Brésil, $\delta^{18} \mathrm{O}=+9,0 \pm 0,1 \%$ ) et de Morafeno (Madagascar, $\delta^{18} \mathrm{O}=+8,9 \pm 0,1 \%$ ) ainsi que

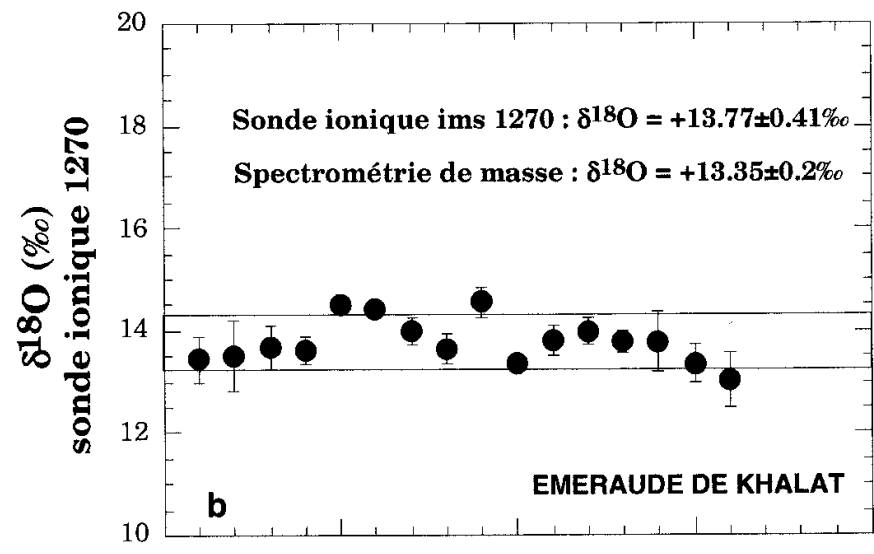

Figure 1b. Reproductibilité des mesures avec la sonde ionique IMS 1270 des compositions isotopiques de l'oxygène de l'émeraude de Khalat (Afghanistan).

celles des Monts Oural (Malysheva : $\delta^{18} \mathrm{O}=+10,6 \pm 0,2 \%$ ) et de Socotó (Brésil, $\delta^{18} \mathrm{O}=+10,6 \%$ ) ont des rapports ${ }^{18} \mathrm{O} /{ }^{16} \mathrm{O}$ voisins qui ne permettent pas de les authentifier.

\section{Application archéologique}

L'intérêt de l'homme pour l'émeraude remonte à l'antiquité [3]. Les premières émeraudes ont été extraites des mines egyptiennes du Djebel Sikaït, vers 1500 avant JC. Pline l'Ancien (23-79 après JC) reprenant les données de Théophraste, signale dans son livre «Histoire Naturelle », trois types d'émeraudes celles de Coptos (Egypte), Scythie (Oural) et Bactriane (actuel Afghanistan). À l'époque romaine, les anciennes mines celtes d'Habachtal (Autriche) et les mines pharaoniques sont exploitées mais définitivement oubliées au Moyen âge. À partir du XVI ${ }^{\text {ème }}$ siècle, les gisements de la Nouvelle Grenade sont exploités par la soldatesque espagnole et la route de l'émeraude suit une piste orientale depuis la Colombie jusqu'en Espagne, puis les cours royales du Nord de l'Europe et enfin, les pays du Moyen Orient et d'Asie. L'époque moghole (1500-1857) sera une étape resplendissante pour la magnification de l'émeraude. Cette pierre, symbole d'immortalité, s'accumulera dans les trésors anciens qui seront pillés et transférés du Caire à Istanbul en 1517, de Delhi à Téhéran en 1739. Le concept des émeraudes dites «vieilles mines» (« old mines ») naît alors [3] et les gemmologues se posent toujours la question de la localisation géographique de ces vieilles mines : les mines afghanes de Bactriane (vallée du Panjshir) étaient-elles déjà exploitées à l'époque d'Alexandre le Grand en 330 avant JC ? ou seulement (re-) découvertes par les soviétiques en 1976 ? Quelle est la provenance des émeraudes accumulées depuis le XVI ${ }^{\mathrm{ème}}$ siècle dans les trésors des musées d'Istanbul, Téhéran, Le Caire, Delhi, Moscou, Saint-Petersbourg, Washington, NewYork, Toronto, Vienne, Paris, Tolède, Londres, Rome ? De Colombie, d'Egypte, d'Autriche, d'Afghanistan, de Russie, d'Inde ou du Pakistan? 


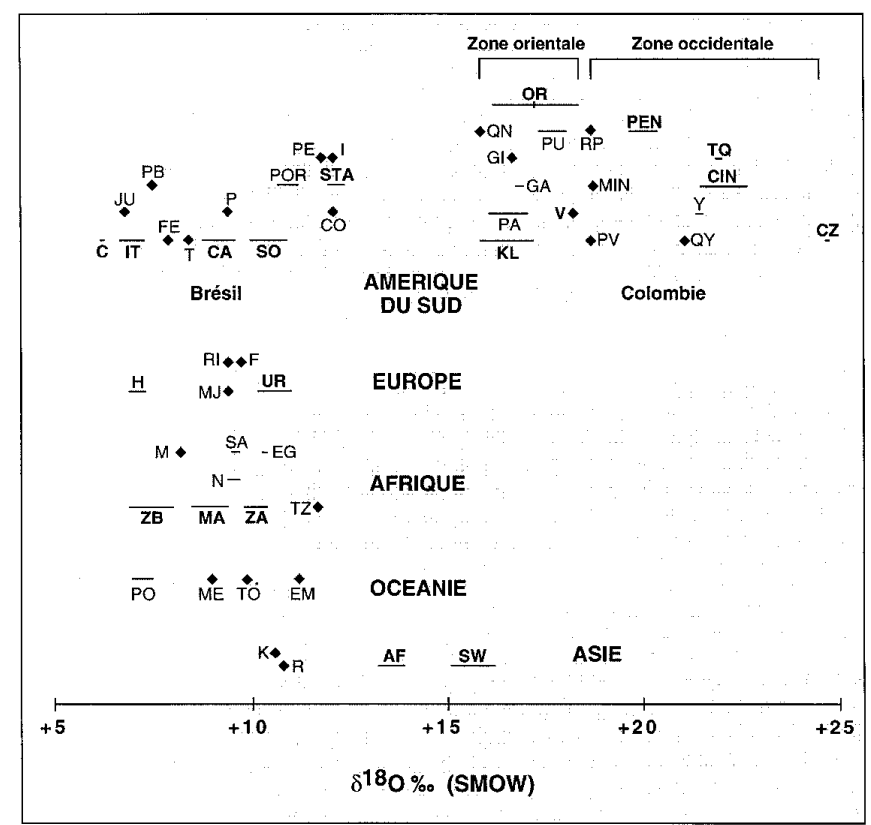

Figure 2. Intervalles de valeur des $\delta^{18} \mathrm{O}(\% \circ \mathrm{SMOW})$ obtenus sur différents indices et gisements d'émeraude dans le monde. Les gisements économiques sont indiqués en caractères gras sur la figure. Asie : Pakistan - Swat (SW), Khaltaro (K) ; Afghanistan - Panjshir (AF) ; Inde - Rajhastan (R). Océanie : Australie - Poona (PO), Menzies (ME), Emmaville (EM), Torrington (TO). Afrique : Egypte - Djebel Sikaït (EG) ; Nigeria - Kaduna (N) ; Tanzanie Sumbawanga (TZ) ; Zambie - Miku (ZA) ; Mozambique - Morrua (M) ; Zimbabwe - Sandawana (ZB) ; Afrique du Sud - Leydsdorp, Cobra pit (SA) ; Madagascar - Morafeno (MA). Europe : Bulgarie - Rila (RI), Norvège - Eidswoll-Mjosa-See (MJ) ; Autriche Habachtal (H) ; Espagne - Franqueira (F), Russie - Oural, Malysheva (UR). Amérique du Sud : Brésil - Quadrilatère ferrifère avec les gisements de Capoeirana (C), Itabira-Belmont (IT) et Santana dos Ferros (FE) ; Tauá (T) ; Carnaíba (CA) ; Socotó (SO) ; Anagé avec les mines de Juca (JU) et Pombos (PB) ; Pirenópolis (P) ; Coqui (CO) ; Porangatu (POR) ; Santa Terezinha de Goiás (STA) ; Pela Ema (PE) ; Itaberaí (I) ; Colombie - zone occidentale : District minier de Muzo avec les mines de Tequendama (TQ), Minabuco (MIN), Repolal (RP), Pava (PV) et Cincho (CIN) ; Coscuez (CZ) ; Yacopi (Y) ; Peña Blanca (PEN), Quaquimay $(Q Y)$ - zone orientale : District minier de Chivor avec les mines d'Oriente (OR), Klein (KL), La Guala (GA), El Pulpito (PU), Palo Aranado (PA), Guali (GI), Quebrada Negra (QN) ; District minier de Gachalá avec la mine de Vega San Juan (V).
Les valeurs des rapports isotopiques ${ }^{18} \mathrm{O} /{ }^{16} \mathrm{O}$ de la plupart des émeraudes, notamment celles qui peuvent appartenir aux émeraudes dites des «vieilles mines » et qui doivent se trouver dans les trésors anciens, sont très contrastées et les xintervalles de valeurs isotopiques distincts (Fig. 2). Ainsi, le rapport isotopique ${ }^{18} \mathrm{O} /{ }^{16} \mathrm{O}$ peut être utilisé comme une propriété exclusive d'authentification. Désormais, l'origine des émeraudes des trésors anciens et la définition de la route de l'émeraude au cours des siècles, dépend de l'analyse isotopique à la sonde ionique IMS 1270.

\section{Conclusion}

L'analyse des isotopes stables de l'oxygène par spectrométrie de masse est une méthode quantitative et destructive qui a conduit à l'élaboration d'une carte isotopique mondiale de l'émeraude. Les rapports isotopiques permettent d'identifier l'origine de la plupart des émeraudes antiques et celles de qualité supérieure rencontrées sur le marché international. Grâce à la technique destructive, nous avons pu calibrer l'analyse isotopique de l'oxygène sur une sonde ionique IMS 1270. Cette technique permet d'analyser directement la surface d'une gemme en ne produisant qu'un minuscule cratère de quelques microns de diamètre et de quelques dizaines d'angström de profondeur. Compte tenu des limites du pouvoir séparateur de l'oeil, cette méthode non destructive est applicable à la gemmologie et à l'archéologie de l'émeraude. Contribution C.R.P.G. No. 1367.

\section{Références}

1. Dossier La gemmologie Analusis 1995, 23(1), 9-54.

2. Giuliani, G.; France-Lanord, C.; Coget, P.; Schwarz, D.; Cheilletz, A.; Branquet, Y.; Giard, D.; Pavel, A.; Martin-Izard, A.; Piat, D. H. Mineralium Deposita 1998, 33, 513-519.

3. Giard, D.; Giuliani, G.; Cheilletz, A.; Fritsch, E.; Gonthier, E. L'émeraude. Connaissances actuelles et prospectives (AFG, CNRS, ORSTOM, Eds.), Paris, p 233.

4. Clayton, R. N.; Onuma, N.; Mayeda, T. K. Earth Planetary Sci. Lett. 1976, 30, 10-18.

5. Clayton, R. N.; Mayeda, T. K. Geochim. Cosmochim. Acta 1963, 27, 43-52. 